PROCEEDINGS OF THE

AMERICAN MATHEMATICAL SOCIETY

Volume 127, Number 4, April 1999, Pages 1011-1020

S 0002-9939(99)04928-X

\title{
ON THE EXACTNESS OF AN S-SHAPED BIFURCATION CURVE
}

\author{
PHILIP KORMAN AND YI LI
}

(Communicated by Hal L. Smith)

\begin{abstract}
For a class of two-point boundary value problems we prove exactness of an S-shaped bifurcation curve. Our result applies to a problem from combustion theory, which involves nonlinearities like $e^{a u /(u+a)}$ for $a>0$.
\end{abstract}

\section{INTRODUCTION}

We consider positive solutions of

$$
u^{\prime \prime}+\lambda f(u)=0 \text { on }(-1,1), u(-1)=u(1)=0 .
$$

Here $\lambda$ is a positive parameter, and we wish to describe all solutions of (1.1) for all values of $\lambda$. Our main example will be $f(u)=e^{\frac{a u}{u+a}}$. This nonlinearity is connected with the steady state of gas combustion according to the Arhenius law (see e.g. J. Bebernes and D. Eberly [2]), and has been studied before (see K.J. Brown, M.M.A. Ibrahim and R. Shivaji [3], R. Shivaji [10] and A. Castro and R. Shivaji [4]). For the above nonlinearity it was shown that for $a \leq 4$ there exists a unique positive solution for all $\lambda$, while for $a$ large the solution diagram is roughly S-shaped, i.e. there is a range of $\lambda$ for which there exist at least three solutions. Moreover, uniqueness of solution was proved for small and for large $\lambda$. In this paper we show that the solution diagram consists of exactly one curve, which is exactly S-shaped, for a class of nonlinearities which includes the one above for $a>a_{0}$, where $a_{0}$ is defined below $\left(a_{0} \simeq 4.35\right)$. The bifurcation diagram is given in Figure 1(a). A similar result was proved by S.-H. Wang [11] using the quadrature technique. In addition to obtaining an alternative proof, we do not require the boundness of $f(u)$, as was the case in [11]. This brings up a possibility of another type of S-shaped solution curves, as in Figure 1(b). Moreover, when verifying the conditions of the theorem for $f(u)=e^{\frac{a u}{u+a}}$, we introduce another technical improvement, which shortens the proof and produces a better critical constant.

Among recent contributions to S-shaped bifurcations, we mention N. Mizoguchi and T. Suzuki [9], and Y. Du and Y. Lou [7], where S-shaped bifurcation occurred in the predator-prey model.

We use tools from bifurcation theory, particularly the Crandall-Rabinowitz bifurcation theorem, which is recalled below, and the techniques from P. Korman, Y. $\mathrm{Li}$ and T. Ouyang [8].

Received by the editors July 8, 1997.

1991 Mathematics Subject Classification. Primary 34B15.

Key words and phrases. S-shaped bifurcation curve, Crandall-Rabinowitz theorem. 


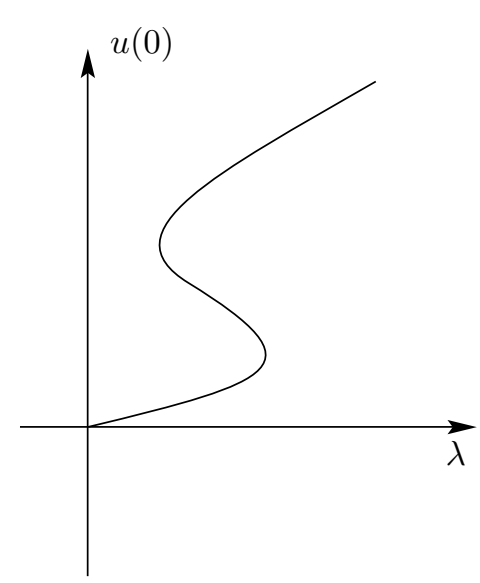

(a)

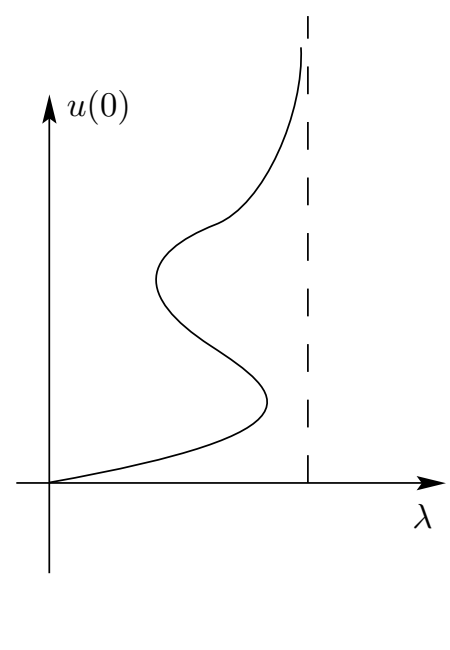

(b)

Figure 1. Two types of S-shaped solution curves

We assume that $f(u) \in C^{2}[0, \bar{u}]$ for some $0<\bar{u} \leq \infty$ and that it satisfies

$$
f(u)>0 \text { for all } 0 \leq u<\bar{u}
$$

also there is $\alpha \in(0, \bar{u})$ such that

$$
f^{\prime \prime}(u)>0 \text { for } u \in(0, \alpha), f^{\prime \prime}(u)<0 \text { for } u \in(\alpha, \bar{u}) .
$$

We also assume that $f(u)$ is "sublinear", i.e. either $\bar{u}$ is finite and $f(\bar{u})=0$, or else

$$
\lim _{u \rightarrow \infty} \frac{f(u)}{u}=0 .
$$

Remark. We denote $F(u)=\int_{0}^{u} f(t) d t$. If $u(x)$ is a positive solution of (1.1), then necessarily $F(u(0))>0$ and $f(u(0))>0$. Therefore, if condition $f(\bar{u})=0$ holds, then all possible values of $u(0)$ must lie in a single interval $(0, \bar{u})$.

For $f(u)=e^{\frac{a u}{u+a}}$ one computes $\alpha=\frac{1}{2} a^{2}-a$. In Theorem 2.1 below we make a further assumption on $f(u)$, which in particular implies that it is "sufficiently convex" in the beginning.

Next we recall the bifurcation theorem of Crandall and Rabinowitz [5].

Theorem 1.1 ([5]). Let $X$ and $Y$ be Banach spaces. Let $(\bar{\lambda}, \bar{x}) \in \mathbf{R} \times X$ and let $F$ be a continuously differentiable mapping of an open neighborhood of $(\bar{\lambda}, \bar{x})$ into $Y$. Let the nullspace $N\left(F_{x}(\bar{\lambda}, \bar{x})\right)=\operatorname{span}\left\{x_{0}\right\}$ be one-dimensional and codim $R\left(F_{x}(\bar{\lambda}, \bar{x})\right)$ $=1$. Let $F_{\lambda}(\bar{\lambda}, \bar{x}) \notin R\left(F_{x}(\bar{\lambda}, \bar{x})\right)$. If $Z$ is a complement of span $\left\{x_{0}\right\}$ in $X$, then the solutions of $F(\lambda, x)=F(\bar{\lambda}, \bar{x})$ near $(\bar{\lambda}, \bar{x})$ form a curve

$$
(\lambda(s), x(s))=\left(\bar{\lambda}+\tau(s), \bar{x}+s x_{0}+z(s)\right),
$$

where $s \rightarrow(\tau(s), z(s)) \in \mathbf{R} \times Z$ is a continuously differentiable function near $s=0$ and $\tau(0)=\tau^{\prime}(0)=0, z(0)=0$, and $z^{\prime}(0)=0$.

We shall write $u(x, \lambda)$ to denote solutions of (1.1). Notice that taking the interval $(-1,1)$ does not restrict the generality for the autonomous equation (1.1). 


\section{The global SOlution CURVE}

We consider positive solutions of

$$
u^{\prime \prime}+\lambda f(u)=0 \text { on }(-1,1), u(-1)=u(1)=0 .
$$

Our main example will be $f(u)=e^{\frac{a u}{u+a}}$. Here $a$ is a fixed positive constant, and $\lambda$ a positive parameter. We wish to describe all positive solutions of (2.1) as the parameter $\lambda$ varies.

The linearized equation corresponding to $(2.1)$ is

$$
w^{\prime \prime}+\lambda f^{\prime}(u) w=0 \text { on }(-1,1), w(-1)=w(1)=0 .
$$

If (2.2) has nontrivial solutions at some solution $u(x)$ of $(2.1)$, we refer to $(\lambda, u)$ as a critical point of (2.1).

Assume there is a $\beta \geq \alpha$ such that

$$
f^{2}(\beta)-2 F(\beta) f^{\prime}(\beta)>0 .
$$

The following lemma is an adaptation of Lemma 2.5 in [8].

Lemma 2.1. Assume that $f(u)$ satisfies the condition (1.2), (1.3) and (1.4). Let $(\lambda, u)$ be any critical point of $(2.1)$, with $u(0) \geq \beta$, and $w(x)$ is the corresponding solution of (2.2). Then

$$
\int_{0}^{1} f^{\prime \prime}(u) u_{x} w^{2} d x>0
$$

Proof. We shall derive a convenient expression for the integral in (2.4). Differentiating (2.2) gives

$$
w_{x}^{\prime \prime}+\lambda f^{\prime}(u) w_{x}+\lambda f^{\prime \prime}(u) u_{x} w=0 .
$$

From equations (2.2) and (2.5)

$$
\left(w w_{x}^{\prime}-w^{\prime 2}\right)^{\prime}+\lambda f^{\prime \prime}(u) u_{x} w^{2}=0
$$

Integrating, we express

$$
\begin{aligned}
\lambda \int_{0}^{1} f^{\prime \prime}(u) u_{x} w^{2} d x & =-\left.\left(w w^{\prime \prime}-{w^{\prime}}^{2}\right)\right|_{0} ^{1} \\
& =w^{\prime 2}(1)-\lambda w^{2}(0) f^{\prime}(u(0)) .
\end{aligned}
$$

Differentiating (2.1) gives

$$
u_{x}^{\prime \prime}+\lambda f^{\prime}(u) u_{x}=0
$$

From $(2.2)$ and $(2.7)$

$$
\left(w u^{\prime \prime}-u^{\prime} w^{\prime}\right)^{\prime}=0
$$

This means that the quantity $w u^{\prime \prime}-u^{\prime} w^{\prime}$ is constant over $[-1,1]$. Evaluating it at $x=0$, we conclude that

$$
w(x) u^{\prime \prime}(x)-u^{\prime}(x) w^{\prime}(x)=-\lambda w(0) f(u(0)) \text { for all } x \in[-1,1] .
$$

Evaluating this expression at $x=1$ gives

$$
w^{\prime}(1)=\frac{\lambda w(0) f(u(0))}{u^{\prime}(1)} .
$$

Multiplying (2.1) by $u^{\prime}$, and integrating over $(0, x)$, we obtain

$$
u^{\prime 2}(x)=2 \lambda[F(u(0))-F(u(x))] .
$$


Setting here $x=1$, and using the resulting formula in (2.8), we express

$$
w^{\prime 2}(1)=\frac{\lambda w^{2}(0) f^{2}(u(0))}{2 F(u(0))} .
$$

Using this in (2.6) we finally obtain

$$
\int_{0}^{1} f^{\prime \prime}(u) u_{x} w^{2} d x=\frac{w^{2}(0)}{2 F(\rho)} I(\rho),
$$

where we denote $\rho=u(0)$, and

$$
I(\rho)=f^{2}(\rho)-2 F(\rho) f^{\prime}(\rho) .
$$

To prove the lemma we need to show that $I(\rho)>0$ for any $\rho \geq \beta$. Compute

$$
I^{\prime}(\rho)=-2 F(\rho) f^{\prime \prime}(\rho) \geq 0 \text { for } \rho \geq \beta,
$$

and the lemma follows by the assumption (2.3).

In order to prove our main result we need to understand precisely how the solution curve changes its direction, which, as it turns out, is determined by the function

$$
h(u)=2 F(u)-u f(u) .
$$

We state our main result next.

Theorem 2.1. Assume that $f(u)$ satisfies the conditions (1.2), (1.3) and (1.4). With $h(u) \equiv 2 F(u)-u f(u)$ assume that

$$
h(\alpha)<0 .
$$

Then the solution curve of (2.1) is exactly $S$-shaped, i.e. it starts at $\lambda=0, u=0$, it makes exactly two turns, and then continues for all $\lambda>0$ without any more turns.

Proof. By the implicit function theorem there is a curve of positive solutions of (2.1) starting at $\lambda=0, u=0$. This curve continues for increasing $\lambda$ until a possible singular solution $\left(\lambda_{0}, u\right)$ (i.e. (2.2) has nontrivial solutions), at which the CrandallRabinowitz Theorem 1.1 applies. A standard calculation shows that the function $\tau(s)$ defined in that theorem satisfies

$$
\tau^{\prime \prime}(0)=-\lambda_{0} \frac{\int_{0}^{1} f^{\prime \prime}(u) w^{3} d x}{\int_{0}^{1} f(u) w d x} .
$$

Indeed, differentiating the equation (2.1) twice in $s$, we have

$$
u_{s s}^{\prime \prime}+\lambda f^{\prime}(u) u_{s s}+2 \lambda^{\prime} f^{\prime}(u) u_{s}+\lambda f^{\prime \prime}(u) u_{s}^{2}+\lambda^{\prime \prime} f(u)=0 .
$$

Setting here $s=0$, and using that $\tau^{\prime}(0)=0$ and $\left.u_{s}\right|_{s=0}=w(x)$, we get

$$
u_{s s}^{\prime \prime}+\lambda_{0} f^{\prime}(u) u_{s s}+\lambda_{0} f^{\prime \prime}(u) w^{2}+\tau^{\prime \prime}(0) f(u)=0 .
$$

Multiplying (2.12) by $w$, and the equation (2.2) by $u_{s s}$, subtracting and integrating, we obtain (2.11). In P. Korman, Y. Li and T. Ouyang [8] it was shown that one may assume $w(x)$ to be positive on $(-1,1)$, and that the denominator in $(2.11)$ is positive. It follows by $(2.11)$ that when $u(0)<\alpha$, only turns to the left in the $(\lambda, u)$ "plane" are possible. Next we need a formula that gives the direction in which the 
solution curve travels, derived in K.J. Brown, M.M.A. Ibrahim and R. Shivaji [3]. With $\rho=u(0)$ and $h(u)$ as defined previously, they show that

$$
\frac{d}{d \rho} \lambda(\rho)^{1 / 2}=\frac{1}{\sqrt{2}} \int_{0}^{1} \frac{h(\rho)-h(\rho v)}{[F(\rho)-F(\rho v)]^{3 / 2}} d v .
$$

We see that $\frac{d \lambda}{d \rho}<0$ and the curve travels to the left, provided

$$
h(\rho)<h(s) \text { for all } s \in(0, \rho) .
$$

We now discuss the function $h(u)$. Since

$$
h^{\prime}(u)=f(u)-u f^{\prime}, \quad h^{\prime \prime}(u)=-u f^{\prime \prime},
$$

it follows that the function $h^{\prime}(u)$ is decreasing on $(0, \alpha)$ and increasing on $(\alpha, \infty)$. Since $h(0)=0, h^{\prime}(0)=f(0)>0$, it follows that there exist $u_{1}$ and $u_{2}$, with $u_{1}<\alpha<u_{2}$, such that $h^{\prime}\left(u_{1}\right)=h^{\prime}\left(u_{2}\right)=0$ and

$$
\begin{gathered}
h^{\prime}=f(u)-u f^{\prime}(u)>0 \text { for } u \in\left(0, u_{1}\right) \cup\left(u_{2}, \infty\right), \\
h^{\prime}=f(u)-u f^{\prime}(u)<0 \text { for } u \in\left(u_{1}, u_{2}\right) .
\end{gathered}
$$

Indeed, existence of the first root $u_{1}<\alpha$ follows immediately by (2.10). As for the second root $u_{2}$, if it did not exist, we would have

$$
f(u)<u f^{\prime}(u) \text { for all } u>\alpha .
$$

Integrating (2.17), we would have

$$
f(u)>\frac{f(\alpha)}{\alpha} u \text { for all } u>\alpha,
$$

contradicting the assumption (1.4), in the case $\bar{u}=\infty$. If $\bar{u}<\infty$, then we get a contradiction by plugging $\bar{u}$ into this inequality. Returning to the function $h(u)$, we notice that $h(0)=0$ and $h(u)$ is concave on $(0, \alpha)$. By $(2.15) h(u)$ is decreasing on $\left(\alpha, u_{2}\right)$, at $u_{2}$ it takes its absolute minimum, and then increases for all $u>u_{2}$. The graph of $h(u)$ is given by Figure 2. One sees that the condition (2.14) holds for all $u \in\left(\alpha, u_{2}\right)$, i.e. as long as $\alpha<u(0, \lambda)<u_{2}$ the solution curve travels to the left.

We now claim that for $\beta=u_{2}$ the condition (2.3) holds. Indeed, since $h\left(u_{2}\right)<0$, we have

$$
f\left(u_{2}\right) u_{2}>2 F\left(u_{2}\right)
$$

Hence

$$
f^{2}\left(u_{2}\right)-2 F\left(u_{2}\right) f^{\prime}\left(u_{2}\right)>f^{2}\left(u_{2}\right)-f\left(u_{2}\right) u_{2} f^{\prime}\left(u_{2}\right)=0,
$$

and the claim follows.

We conclude that for $u(0)>u_{2}$ Lemma 2.1 applies. We show next that this implies that only turns to the right in the $(\lambda, u)$ plane are possible. We proceed similarly to P. Korman, Y. Li and T. Ouyang [8]. Let $\left(\lambda_{0}, u_{0}(x)\right)$ be a critical point of $(2.1)$ with $u_{0}(0)>u_{2}$. The function $f^{\prime \prime}\left(u_{0}(x)\right)$ changes sign exactly once on $(0,1)$, say at $x_{0}>0$. Clearly

$$
f^{\prime \prime}\left(u_{0}(x)\right)<0 \text { for } x \in\left(0, x_{0}\right), f^{\prime \prime}\left(u_{0}(x)\right)>0 \text { for } x \in\left(x_{0}, 1\right) .
$$

By stretching the function $w(x)$ we may assume that the functions $w(x)$ and $-u_{0}^{\prime}(x)$ intersect at $x_{0}$. We claim that $x_{0}$ is the only point on $(0,1)$ where they intersect. Indeed, the functions $w(x)$ and $-u_{0}^{\prime}$ are solutions of the same linear equation (2.2). 


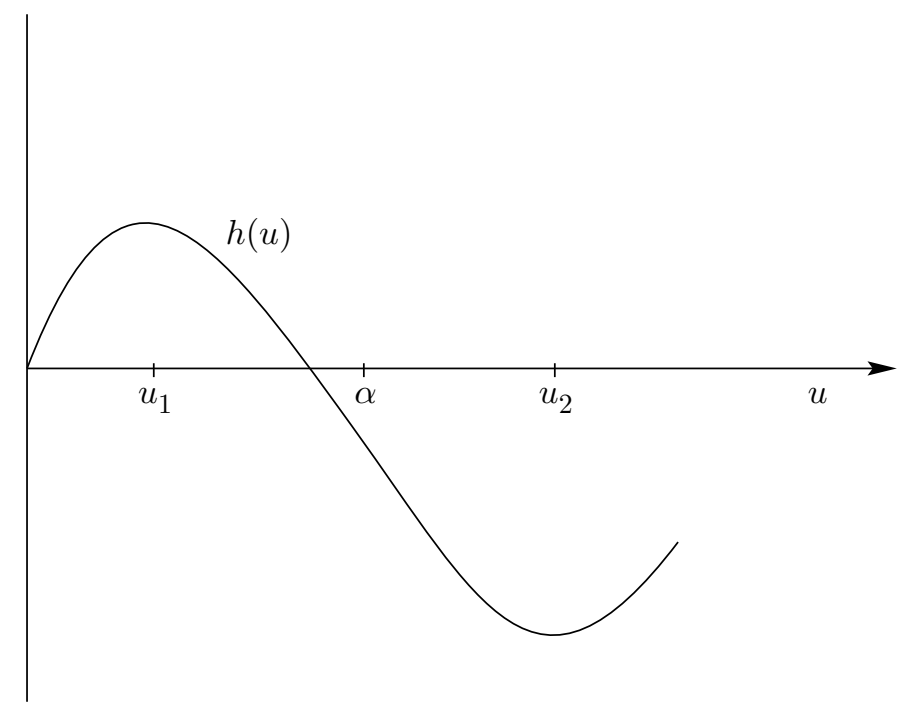

Figure 2. The function $h(u)$

If $x_{1}$ is another intersection point, adjacent to $x_{0}$, then we can find a constant $\mu \neq 1$ and a point $\bar{x} \in\left(x_{0}, x_{1}\right)$ so that $\mu w(\bar{x})=-u_{0}(\bar{x})$ and $\mu w^{\prime}(\bar{x})=-u_{0}^{\prime}(\bar{x})$, i.e. two distinct solutions with the same initial conditions, a contradiction. By Lemma 2.1 it follows that

$$
\int_{0}^{1} f^{\prime \prime}\left(u_{0}(x)\right) w^{2} w d x<\int_{0}^{1} f^{\prime \prime}\left(u_{0}(x)\right) w^{2}\left(-u_{x}\right) d x<0 .
$$

Indeed, $-u_{x}>w$ where $f^{\prime \prime}>0$, and $-u_{x}<w$ where $f^{\prime \prime}<0$. Hence the integrand on the right is pointwise greater than the one on the left. By (2.19) the numerator in (2.11) is negative, which means that only turns to the right are possible for $u(0)>u_{2}$.

We now return to the curve of solutions, which started at $\lambda=0, u=0$. It is well-known that $u(0, \lambda)$ is increasing on this curve for all $\lambda$; see e.g. E.N. Dancer [6]. By the time $u(0, \lambda)$ reaches $\alpha$, the curve already travels to the left. Since $f^{\prime \prime}(u)>0$ for $u<\alpha$, it follows by $(2.11)$ that only turns to the left are possible before $u(0, \lambda)$ reaches $\alpha$, and hence exactly one such turn occurred. As $u(0, \lambda)$ keeps increasing between $\alpha$ and $u_{2}$, the function $h(u)$ keeps decreasing, and hence (2.14) continues to hold, and so the solution curve keeps travelling to the left until $u(0, \lambda)$ reaches $u_{2}$. When $u(0, \lambda)>u_{2}$, only turns to the right are possible, and indeed exactly one such turn will occur, for if the curve kept travelling to the left it would have nowhere to go (solutions of (2.1) are bounded for bounded $\lambda$ ). Hence the solution curve is exactly S-shaped.

Finally, there is only one solution curve, since (in case $f(u)>0$ for all $u$ ) on our curve of solutions, the value $u(0)$ varies from 0 to $\infty$, while the value of $u(0)$ uniquely identifies the solution; see e.g., E.N. Dancer [6]. (In the case where $f(u)$ vanishes at some $\bar{u}$, one argues similarly.) 
Next we discuss a generalization of Theorem 2.1, obtained by replacing the sublinearity condition (1.4) by the following "weak sublinearity condition"

$$
\left(\frac{f(u)}{u}\right)^{\prime} \leq 0 \text { for some } u=u_{0}>\alpha .
$$

Theorem 2.2. Assume that $f(u)$ satisfies the conditions (1.2), (1.3) and (2.20). Then the solution curve of (2.1) is either exactly S-shaped as described in the previous theorem, or else after exactly two turns it tends to infinity at some finite $\bar{\lambda}>0$.

Proof. We begin by observing that our conditions allow $f(u)$ to be asymptotically linear as $u \rightarrow \infty$. Indeed, starting with any $f(u)$ satisfying (1.2) and (1.3), we may, by adding a large constant, obtain a function satisfying the condition (2.20) as well, at any $u_{0}>\alpha$. This leaves $f(u)$ free to have any behavior at infinity, which is consistent with concavity, in particular it can be asymptotically linear. This implies that the solution curve may go to infinity at some finite $\lambda=\bar{\lambda}$.

The proof proceeds similarly to the previous theorem. When it comes to the existence of $u_{2}$, the second root of $h(u)$, we observe that if it did not exist, we would have

$$
f(u)<u f^{\prime}(u) \text { for all } u>\alpha,
$$

contradicting the assumption (2.20). As before, the curve makes precisely one turn before $u(0)$ reaches $\alpha$. We claim that the curve cannot go to infinity as it travels to the left. Indeed, setting $w(u)=\left(\frac{f(u)}{u}\right)^{\prime}$, we observe that by $(2.20) w\left(u_{0}\right) \leq 0$. Since

$$
w^{\prime}(u)+\frac{2}{u} w(u)=\frac{f^{\prime \prime}(u)}{u}<0,
$$

we obtain by integrating the above equation

$$
u^{2} w(u)=u_{0}^{2} w\left(u_{0}\right)+\int_{u_{0}}^{u} s f^{\prime \prime}(s) d s<0 \text { for all } u \geq u_{0} .
$$

We conclude that $\frac{f(u)}{u}$ is decreasing for all $u>u_{0}$. Hence if $f(u)$ is asymptotical to $a u+b$, with constants $a$ and $b$, then $b>0$. This implies that bifurcation from infinity is to the left; see e.g. [1]. We conclude that the solution curve cannot go to infinity on its way to the left.

Hence as before the curve will make exactly one more turn to the right, and then it may go to infinity at some finite $\bar{\lambda}$, see Figure $1(\mathrm{~b})$.

\section{Applications}

As our first application, we now consider $f(u)=e^{\frac{a u}{u+a}}$. Then $f^{\prime}(u)>0$ for all $u>0, f^{\prime \prime}(u)>0$ for $0<u<\frac{1}{2} a(a-2), f^{\prime \prime}(u)<0$ for $u>\frac{1}{2} a(a-2)$. If $0<a \leq 4$, it was noticed previously (see e.g. R. Shivaji [10]) that $f(u)>u f^{\prime}(u)$ for all $u>0$, and hence for any $\lambda$ the problem (2.1) has a unique solution. (The previous writers were using the formula (2.13) to conclude uniqueness in this case. Alternatively, one could use the Sturm comparison theorem to conclude that the linearized equation (2.2) can have no nontrivial solutions, and so the solution curve cannot turn.) 
Next we need the following lemma.

Lemma 3.1. Let $a_{0}>4$ be a solution of

$$
1-\frac{2}{a}=4 \int_{2}^{a} \frac{e^{-\tau+2}}{\tau^{2}} d \tau
$$

(Numerical evaluation shows that $a_{0} \simeq 4.35$.) Then in the case when $f(u)=e^{\frac{a u}{u+a}}$ we have for all $a>a_{0}$

$$
h(\alpha)<0 .
$$

Proof. First of all notice that for all $a>a_{0}$

$$
1-\frac{2}{a}>4 \int_{2}^{a} \frac{e^{-\tau+2}}{\tau^{2}} d \tau
$$

Indeed, denoting by $d(a)$ the difference between the left and right sides in (3.3), we have that $d\left(a_{0}\right)=0$ and

$$
d^{\prime}(a)=\frac{2}{a^{2}}-4 \frac{e^{-a+2}}{a^{2}}>0 \text { for } a>4 .
$$

This justifies (3.3) and also shows that the equation (3.1) has exactly one solution in the $a>4$ range. Compute

$$
h(\alpha)=2 \int_{0}^{\frac{1}{2} a^{2}-a} e^{\frac{a s}{s+a}} d s-\left(\frac{1}{2} a^{2}-a\right) e^{a-2} \equiv-\frac{e^{a-2} a^{2}}{2} H,
$$

where

$$
\begin{aligned}
H & =1-\frac{2}{a}-\frac{4}{a^{2}} \int_{0}^{\frac{1}{2} a^{2}-a} e^{\frac{a s}{s+a}-a+2} d s \\
& =1-\frac{2}{a}-\frac{4}{a^{2}} \int_{0}^{\frac{1}{2} a^{2}-a} e^{-\frac{a^{2}}{a+s}+2} d s .
\end{aligned}
$$

We make a change of variables $\tau=\frac{a^{2}}{a+s}$, i.e. $s=\frac{a^{2}}{\tau}-a, d s=-\frac{a^{2}}{\tau^{2}} d \tau$. It follows that

$$
H=1-\frac{2}{a}+4 \int_{a}^{2} \frac{e^{-\tau+2}}{\tau^{2}}>0
$$

in view of $(3.3)$, and hence $h(\alpha)<0$.

Remark. If the reader is uncomfortable with a (routine) use of a computer to solve the equation (3.1), one can proceed as follows. In (3.4) set $t=\tau-2$. Then

$$
\begin{aligned}
H & =1-\frac{2}{a}-4 \int_{0}^{a-2} \frac{e^{-t}}{(t+2)^{2}} d t \\
& =1-\frac{2}{a}-4 \int_{0}^{a-2} e^{-t}\left[\frac{1}{(t+2)^{2}}-\frac{1}{4}\right] d t-\int_{0}^{a-2} e^{-t} d t \\
& =-\frac{2}{a}+e^{-a+2}+\int_{0}^{a-2} e^{-t} \frac{4 t+t^{2}}{(t+2)^{2}} d t,
\end{aligned}
$$

which is positive for large $a$. 
If $a>4$, then $h^{\prime}(u)$ has roots and in fact, $u_{1}, u_{2}=\frac{1}{2} a^{2}-a \pm \frac{a}{2} \sqrt{a^{2}-4 a}$. If $a>a_{0} \simeq 4.35$, then by Lemma 3.1 the condition (2.10) is satisfied, and hence the solution curve is exactly $\mathrm{S}$-shaped. This is an improvement of the critical constant $a_{0} \simeq 4.4967$ obtained by S.H. Wang [11]. We have thus proved the following theorem.

Theorem 3.1. If $a>a_{0}$, then the solution curve for the problem

$$
u^{\prime \prime}+\lambda e^{\frac{a u}{u+a}}=0 \quad \text { on }(-1,1), u(-1)=u(1)=0
$$

is exactly S-shaped.

Our second example involves $f(u)=1+u^{2}-\epsilon u^{p}$, with constants $p>2$, and $\epsilon>0$. We compute $h(u)=u-\frac{1}{3} u^{3}+\epsilon \frac{p-1}{p+1} u^{p+1}$, and $\alpha=\left[\frac{2}{\epsilon p(p-1)}\right]^{\frac{1}{p-2}}$. It follows that

$$
h(\alpha)=\left[\frac{2}{\epsilon p(p-1)}\right]^{\frac{1}{p-2}}-\frac{1}{3}\left[\frac{2}{\epsilon p(p-1)}\right]^{\frac{3}{p-2}}+\epsilon \frac{p-1}{p+1}\left[\frac{2}{\epsilon p(p-1)}\right]^{\frac{p+1}{p-2}} .
$$

For $\epsilon$ small the leading terms in $\epsilon$ are second and third, and they have the same order in $\epsilon$. We will have $h(\alpha)<0$ for small $\epsilon$, provided

$$
\frac{1}{3}\left[\frac{2}{p(p-1)}\right]^{\frac{3}{p-2}}>\frac{p-1}{p+1}\left[\frac{2}{p(p-1)}\right]^{\frac{p+1}{p-2}} .
$$

This is equivalent to

$$
\frac{1}{3}>\frac{2}{p(p+1)},
$$

which is true for $p>2$. We conclude that for $\epsilon$ small, i.e. if

$$
\epsilon^{\frac{2}{p-2}}<\frac{1}{3}\left[\frac{2}{p(p-1)}\right]^{\frac{2}{p-2}}-\frac{p-1}{p+1}\left[\frac{2}{p(p-1)}\right]^{\frac{p}{p-2}},
$$

the solution curve is exactly S-shaped.

Theorem 3.2. Assume that $p>2$ and $\epsilon$ satisfies (3.5). Then the solution curve for

$$
u^{\prime \prime}+\lambda\left(1+u^{2}-\epsilon u^{p}\right)=0 \quad \text { on }(-1,1), u(-1)=u(1)=0
$$

is exactly $S$-shaped.

\section{ACKNOWLEDGEMENT}

We wish to thank the Institute for Mathematics and its Applications at the University of Minnesota (IMA) for supporting our visits there, which made this work possible. We thank R. Shivaji for pointing out the reference [11] to us.

\section{REFERENCES}

[1] A. Ambrosetti and P. Hess, Positive solutions of asymptotically linear elliptic eigenvalue problems, J. Math. Anal. Applic. 73, 411-422 (1980). MR 81j:35092

[2] J. Bebernes and D. Eberly, Mathematical Problems from Combustion Theory, SpringerVerlag (1989). MR 91d:35165

[3] K.J. Brown, M.M.A. Ibrahim and R. Shivaji, S-shaped bifurcation curves, Nonlinear Anal. TMA 5 (5), 475-486 (1981). MR 82h:35007

[4] A. Castro and R. Shivaji, Uniqueness of positive solutions for elliptic boundary value problems, Proc. Royal Soc. Edinburgh 98A, 267-269 (1984). MR 86d:35051 
[5] M.G. Crandall and P.H. Rabinowitz, Bifurcation, perturbation of simple eigenvalues and linearized stability, Arch. Rational Mech. Anal. 52, 161-180 (1973). MR 49:5962

[6] E.N. Dancer, On the structure of solutions of an equation in catalysis theory when a parameter is large, J. Differential Equations 37, 404-437 (1980). MR 82b:35018

[7] Y. Du and Y. Lou, S-Shaped global bifurcation curve of positive solutions to a prey-predator model, MSRI Preprint No. 1996-044 (1996).

[8] P. Korman, Y. Li and T. Ouyang, Exact multiplicity results for boundary-value problems with nonlinearities generalizing cubic, Proc. Royal Soc. Edinburgh 126A, 599-616 (1996). MR 97c:34038

[9] N. Mizoguchi and T. Suzuki, Equations of gas combustion: s-shaped bifurcation and mushrooms, J. Differential Equations 134, 183-215 (1997). MR 97m:35090

[10] R. Shivaji, Remarks on an S-shaped bifurcation curve, J. Math. Anal. Applic. 111, 374-387 (1985). MR 87c:35015

[11] S.-H. Wang, On S-shaped bifurcation curves, Nonlinear Analysis TMA 22, 1475-1485 (1994). MR 96d:34021

Department of Mathematical Sciences, University of Cincinnati, Cincinnati, Ohio 45221-0025

E-mail address: kormanp@math.uc.edu

Department of Mathematics, University of Iowa, Iowa City, Iowa 52242

E-mail address: yli@math.uiowa.edu 